\title{
Gynaekologische Gesellschaft Der Deutschen Schweiz
}

Herbstversammluag am 22. November 1953 in Basel

Verzeichnis der Vorträge:

Bírcher, Jost: Zur gezielten Thrombose-Prophylaxe mit Marcoumar

Reíst, A.: Selektive Prophylaxe der thrombo-embolischen Erkrankungen im

Wochenbett mit Tromexan 214

Schlußwort Reist Fries, K.: Versuch einer generellen Thromboseprophylaxc im Wochenbett mit Marcoumar 220

König, F. E., Th. Reich mid M. Steínbrüchel: Thromboseprophylaxe mit Marcoumar im Wochenbett und Zustand des Kindes (erscheint ausführlich an anderer Stelle) 225

Diskussion: Aepplí, Hartert Diskussion zu den Vorträgen 1-4: Held, Merz, Breítner, Glatthaar, Wespi,

Malagomba 227

Wenner, R.: Fehldiagnosen und Fehlbehandlungen bei Sterilitätsfällen (er scheint in extenso an anderer Stelle) 231

Diskussion; Glatthaar Mayer, A.: Bemerkungen zur seelisch bedingten Sterilität (Manuskript nicht eingegangen) 231

Stuckí, D.: Paralysie infantile et Maternité 231

Hauser, A.: Teste mit Ganglíenblocker und Hydrazinophthalazine bei Nephropathie (erscheint in extenso an anderer Stelle) 234

Müller, Carl: Postpartuale endokrine Syndrome und Morbus Sheehan 235 Aepplí, H.: Beziehungen zwischen Schilddrüsen- und Eierstockfunktíon (hierzu Tafel I) 238

Diskussion: Müller Tschumi, Rene: Die Behandlung der genitalen Hypoplasie durch intramurale Ovocyclin-Kristallinjektionen 242

Breítner, J.: Ostrogenausscheidung beim normalen und gestörten Zyklus 245

Berger, Jean und Max Keller: Östrogenwirkung in der Menopause 250

Keller, Max und Andre Hauser: Die Phenolsteroidausscheidung bei Kastratinnen nach ACTH-Belastung 255

Diskussion: Sauter Nigglí, G.: Ein einfaches Gerät zur Wiederbelebung asphyktischer Neugeborener 260

Mayer, A.: Nachteile der reinen Steißlage mit hochgeschlagenen Beinen (Manuskript nicht eingegangen) 264

Guggisberg, W.: Beítrag zur Schmerzlinderung unter der Geburt

Roth, F.: Injektionsbehandlung des Descensus mit Dondren 269

Sauter, H.: Sterilisation post partum von einem Periumbilicalschnitt aus (erscheint ausführlich an anderer Stelle) 